Nervenarzt 2012 $\cdot 83: 1095-1096$

DOI 10.1007/s00115-011-3436-y

Online publiziert: 10. August 2012

(c) Springer-Verlag 2012

H. Gündel ${ }^{1}$ P. Henningsen ${ }^{2}$ - H.P. Kapfhammer ${ }^{3}$ 'W. Rief $^{4}$

${ }^{1}$ Klinik und Poliklinik für Psychosomatische Medizin und Psychotherapie, Universitätsklinikum Ulm

${ }^{2}$ Klinik und Poliklinik für Psychosomatische Medizin und Psychotherapie der

Technischen Universität München, Klinikum rechts der Isar, München

${ }^{3}$ Univ. Klinik für Psychiatrie, Medizinische Universität Graz

${ }^{4}$ Klinische Psychologie und Psychotherapie, Universität Marburg

\title{
Die Störungsgruppe der somatoformen Störungen
}

\section{Alte und neue Herausforderungen}

Die mit dem DSM-III erstmals eingeführte diagnostische Kategorie der „somatoformen Störungen “ begleiten von Anfang an kritische Stimmen. Einerseits wurde mit dieser Neukonzeptualisierung einer bedeutsamen Patientengruppe in der psychiatrischen und psychosomatischen Versorgung ein einheitlicher diagnostischer Ort zugesprochen, von dem aus wichtige epidemiologische, klinisch-diagnostische und therapeutische Forschungsaktivitäten gestartet und koordiniert werden konnten. Andererseits haften der diagnostischen Konstruktion der „somatoformen Störungen“ erhebliche Mängel an. In der Begrifflichkeit „somatoform“ setzt sich eine wissenschaftstheoretisch überholte Dichotomie von „Leib-Seele“ fort. Das Postulat bedingender psychosozialer Stressoren kann empirisch keineswegs immer bestätigt werden und legt einen einseitigen "psychogenetischen" Fokus auf ein komplexes pathogenetisches Geschehen, das vorteilhaft nur innerhalb eines biopsychosozialen Modells beschrieben werden kann. Die Forderung einer „medizinischen Nichtbegründbarkeit“ ist prinzipiell nicht zu verifizieren und für die ärztliche Routine wenig tauglich. Die zentrale Orientierung an einer lediglich aufzusummierenden Anzahl von „medizinisch unerklärten körperlichen Symptomen“ berücksichtigt weder die einzelnen somatoformen Störungen zugrunde liegende psychopathologische Vielschichtigkeit noch die Charakteristika eines bestimmenden Krankheitsver- haltens. Die Konstruktion als exklusiver diagnostischer Kategorie verfehlt die gerade in der ärztlichen Primärversorgung vorherrschende Überschneidung von somatoformen Störungen mit anderen psychischen Störungen wie z. B. Angst- und depressiven Störungen einerseits, mit somatisch-medizinischen Krankheiten andererseits. Es überrascht nicht, dass die mit dem DSM-III erscheinende Diagnosegruppe der „somatoformen Störungen“ im ärztlichen Versorgungsalltag letztlich nicht wirklich angekommen ist und hier weiterhin mit Alternativkonzepten „psychogener" und „funktioneller Störungen“ konkurriert.

\section{》) Bevorstehende Revisionen von DSM-5 und ICD-11 werden zentrale Charakteristika des Krankheitsverhaltens berücksichtigen}

Die bevorstehenden Revisionen von DSM-5 und ICD-11 haben in zahlreichen Task-Forces intensive Diskussionen zur Neukonzeptualisierung der somatoformen Störungsgruppe angestoßen. Bedeutsame Veränderungen werden in ersten vorliegenden Entwürfen erkennbar. In einer einführenden Arbeit zum Schwerpunktthema ,somatoforme Störungen" in diesem Heft bemühen sich Constanze Hausteiner-Wiehle und Peter Henningsen, die komplexen Fragen zur Konzeptualisierung und Diagnostik der somatoformen Störungen aufzunehmen und die sich in künftigen Klassifikationssystemen andeutenden diagnostischen Algorithmen darzustellen. Als eine wesentliche Veränderung zeichnet sich ab, dass künftig zentralen Charakteristika des Krankheitsverhaltens eine gröBere Beachtung geschenkt werden wird. Damit sollen unabhängig von einer letztlich angestrebten psychosomatischen und somatopsychischen Bewertung jene Faktoren in einem umfassenden biopsychosozialen Diagnoseansatz beschrieben werden, die empirisch wesentlich zur funktionellen Beeinträchtigung der Patienten und damit zur Erkrankungsschwere beitragen und oft mit konkretem klinischem Handlungsbedarf verbunden sind.

Michael Noll-Hussong und Harald Gündel machen in ihrem Beitrag klar, wie komplex die zahlreichen, potenziellen ätiopathogenetischen Einflüsse auf die Entstehung, Auslösung und Aufrechterhaltung somatoformer Störungen angenommen werden müssen. Die große Disparität der in den Studien gefundenen und in der wissenschaftlichen Literatur diskutierten Faktoren auf den unterschiedlichen theoretischen und methodischen Untersuchungsebenen verweist auf die Tatsache, dass nach wie vor eine vereinheitlichende Modellbildung schmerzlich vermisst wird.

Japhia-Marie Gottschalk und Winfried Rief präsentieren einen Überblick über psychotherapeutische Ansätze für 
Patienten mit somatoformen Störungen. Sie beschreiben Gründe, warum Ärzte und Psychotherapeuten den Umgang mit dieser Patientengruppe oft so schwierig und herausfordernd erleben. In einem transdiagnostischen Ansatz skizzieren sie grundlegende Abschnitte des therapeutischen Vorgehens, das sich vorteilhaft in den Aufbau einer therapeutischen Beziehung, die Vermittlung eines Störungsmodells und die Vermittlung von Bewältigungsstrategien gliedert. In diese strukturelle Ausrichtung können sowohl kognitiv-verhaltenstherapeutische als auch psychodynamische Konzepte gewinnbringend eingeführt werden, wie eine Zusammenstellung der randomisierten und kontrollierten Studien belegt.

Hans-Peter Kapfhammer führt aus, dass eine biopsychosoziale Konzeptualisierung somatoformer Störungen und funktioneller Körpersyndrome eine Rationale auch für psychopharmakotherapeutische Ansätze begründen kann. Anhand einer Übersicht über randomisierte, doppelblinde und zumeist auch placebokontrollierte Studien werden grundlegende psychopharmakologische Strategien beschrieben.

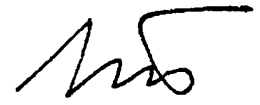

Prof. Dr. Harald Gündel

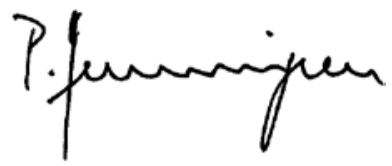

Prof. Dr. Peter Henningsen

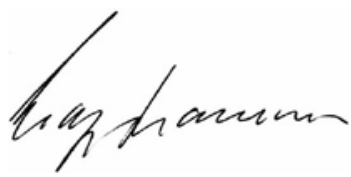

Prof. Dr. Dr. Hans-Peter Kapfhammer

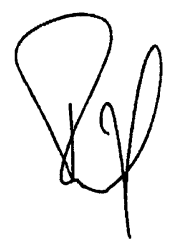

Prof. Dr. Winfried Rief

\section{Korrespondenzadresse}

Prof. Dr. Dr. H.P. Kapfhammer

Univ. Klinik für Psychiatrie,

Medizinische Universität Graz

Auenbruggerplatz 31/1, 8036 Graz

Österreich

hans-peter.kapfhammer@klinikum-graz.at

Interessenkonflikt. Der korrespondierende Autor gibt für sich und seine Koautoren an, dass kein Interessenkonflikt besteht.

\section{Depression bei Männern - genetisch bedingt?}

Männliche Patienten mit bipolarer affektiver Störung zeigten in einer molekulargenetischen Studie des Universitätsklinikums Heidelberg mit 4200 Teilnehmern besonders häufig eine bestimmte genetische Veränderung, betroffene Frauen allerdings nicht. Die untersuchte Genvariante, die zu veränderter Funktion des Serotoninrezeptors Typ 3 führt, erhöht das Erkrankungsrisiko bei Männern um etwa 30\%. Der Rezeptor als eines der Schlüsselmoleküle der neuronalen Kommunikation ist an Prozessen wie Lernen, Erkennen und Emotionen beteiligt. Laut den beteiligten Forschern könnte eine veränderte Signalwirkung des Neurotransmitters Serotonin eine Ursache für die Entstehung von Angststörungen sein, die bei manischer Depression eine große Rolle spielen. Auf Nervenzellen sitzen verschiedene Typen von Rezeptoren, an die das Serotonin nach dem Schlüssel-SchlossPrinzip bindet und so zelluläre Signale weiterleitet. Einer davon ist der Serotoninrezeptor Typ 3. Die Genveränderung stört dieses Wechselspiel zwischen Serotonin und seinem Rezeptor. Dadurch ändern sich die Weiterleitung von Signalen und so die emotionale Verarbeitung von Reizen. Es bleibt aufzuklären, ob die Wirksamkeit der therapeutisch eingesetzten Rezeptor-Blocker von der individuellen Genvariante der Patienten abhängig ist. So könnten in Zukunft Genprofil-spezifische Medikamente entwickelt werden.

Literatur: Hammer C, Cichon S, Niesler B et al (2012) Replication of functional serotonin receptor type $3 A$ and $B$ variants in bipolar affective disorder: a European multicenter study. Translational Psychiatry doi:10.1038/ tp.2012.30

Quelle: Universitätsklinikum Heidelberg, www.klinikum.uni-heidelberg.de 